\title{
34
}

\section{Effective coefficients of thermoconductivity on some symmetric periodically perforated plane structures}

\section{J. Vucans}

Department of Mathematical Analysis, University of Latvia Rainis boulevard 19, Riga, LV-1586, Latvia.

Phone: +371-2-615646. Fax: +371-7-820113. E-mail: fmmak@cclu.lv

\begin{abstract}
In this article we discuss an auxiliary problem which arises in the homogenization theory for the Laplacian on the plane with periodic array of square holes and homogeneous Neumann boundary conditions on those. Independently, this problem describes the process of thermoconductivity. We find the explicit formulas for effective coefficients of thermoconductivity (homogenized modula). We make also the asymptotic analysis of these formulas in the cases of big and small holes.
\end{abstract}

\section{Keywords}

Homogenization, Laplacian, periodic structure, holes, asymptotic analysis

\section{INTRODUCTION}

First let's introduce for the numerical parameters $\gamma$ and $\theta, 0<\gamma<1,0 \leq \theta \leq 1$, the sets

$$
\begin{aligned}
H_{\gamma, \theta}= & \left\{\left(x_{1}, x_{2}\right) \in R^{2} \mid x_{1}=\frac{1}{2}+r \cos \left(\varphi-\theta \frac{\pi}{2}\right), x_{2}=\frac{1}{2}+r \sin \left(\varphi-\theta \frac{\pi}{2}\right),\right. \\
& \left.0 \leq \varphi<2 \pi, \quad 0 \leq r \leq \frac{\gamma}{2 \max \{|\cos \varphi||\sin \varphi|\}}\right\} .
\end{aligned}
$$

For fixed $\gamma$ and $\theta$ the set $H_{\gamma, \theta}$ is a square with the center $(1 / 2,1 / 2)$ and the length of side equal to $\gamma$; the parameter $\theta$ determines its orientation in the plane. We shall concentrate our attention to the two cases $\theta=0$ and $\theta=1$, which correspond to the situations when the sides (for $\theta=0$ ) or the diagonals (for $\theta=1$ ) of the squares $H_{\gamma, \theta}$ are parallel to the coordinate axis. 
Let's introduce also the periodic perforated plane structures

$B_{\gamma, \theta}=R^{2} \backslash \bigcup_{k=-\infty}^{\infty} \bigcup_{j=-\infty}^{\infty}\left((k, j)+H_{\gamma, \theta}\right)$,

their unit cells of periodicity $C_{\gamma, \theta}$ and the quarters $Q_{\gamma, \theta}$ of these unit cells:

$C_{\gamma, \theta}=B_{\gamma, \theta} \cap(-1 / 2,1 / 2)^{2}, \quad Q_{\gamma, \theta}=B_{\gamma, \theta} \cap(0,1 / 2)^{2}$.

Let's for an arbitrary set $S \subset R^{2}$ denote by $\partial S$ the boundary of this set.

This article refers to the homogenization theory for the Dirichlet problem of the Laplacian on the perforated plane structures $B_{\gamma, 0}$ and $B_{\gamma, 1}$ with homogeneous Neumann conditions on the boundaries of holes.

In order to find for such problems (with $\theta=0$ or 1 ) the homogenized matrix, which is due to symmetry equal to $\left(K_{\gamma, \theta} \delta_{i j}\right)$, we have to solve the auxiliary problem on the unit cell $C_{\gamma, \theta}$ :

$$
\begin{array}{lll}
\Delta U_{\gamma, \theta}=0 & \text { in } & C_{\gamma, \theta}, \\
\frac{\partial U_{\gamma, \theta}}{\partial n}=0 & \text { in } & \partial C_{\gamma, \theta} \backslash \partial(-1 / 2,1 / 2)^{2}, \\
\left(U_{\gamma, \theta}-x_{1}\right) & \text { is } & 1 \text {-periodic in both variables } x_{1} \text { and } x_{2},
\end{array}
$$

where $n$ is the unit outward normal of the set $C_{\gamma, \theta}$. The homogenized modula, which in this case is the effective coefficient of thermoconductivity, is equal to

$$
K_{\gamma, \theta}=\frac{1}{m e s C_{\gamma, \theta}} \iint_{C_{\gamma, \theta}} \frac{\partial U_{\gamma, \theta}\left(x_{1}, x_{2}\right)}{\partial x_{1}} d x_{1} d x_{2} .
$$

We note that such problems for more general periodic structures were discussed in the books of Bakhvalov and Panasenko (1989) and Bensoussan, Lions and Papanicolaou (1978). Some results concerning the asymptotic analysis of $K_{\gamma, 0}$ and $K_{\gamma, 1}$ as the functions of the parameter $\gamma$ are obtained in the papers of Kozlov (1989), Kozlov and Vucans (1992) and Vucans (1992). In the present paper, using the method similar as in the article of Kozlov and Vucans (1992), we continue these studies.

\section{FORMULAS FOR EFFECTIVE COEFFICIENTS $K_{\gamma, 0}$ AND $K_{\gamma, 1}$}

In order to formulate the main results of this article we shall use the following functions:

$$
\begin{aligned}
\varphi(t, \alpha) & =\frac{1}{\sqrt{(1+t)\left(\alpha^{2}-t^{2}\right)}}, \\
\psi_{0}(t, \alpha) & =\frac{t}{\left(1-t^{2}\right)\left(\alpha^{2}-t^{2}\right)} \\
I_{10}(\alpha) & =\int_{1}^{\alpha} \sqrt{-\psi_{0}(t, \alpha)} d t
\end{aligned}
$$

$$
\begin{aligned}
& \psi_{1}(t, \beta)=\frac{1}{\left(1-t^{2}\right)\left(\beta^{2}-t^{2}\right)^{2}}, \\
& I_{11}(\beta)=\int_{1}^{\beta} \sqrt[4]{-\psi_{1}(t, \beta)} d t
\end{aligned}
$$




$$
\begin{array}{rlrl}
I_{20}(\alpha) & =\int_{0}^{1} \sqrt{\psi_{0}(t, \alpha)} d t, & I_{21}(\beta) & =\int_{0}^{1} \sqrt[4]{\psi_{1}(t, \beta)} d t \\
I_{30}(\alpha) & =\int_{0}^{1} \sqrt{\psi_{0}(z, \alpha)} \int_{z}^{\alpha} \varphi(t, \alpha) d t d z, & I_{31}(\beta)=\int_{-1}^{1} \sqrt[4]{\psi_{1}(z, \beta)} \int_{z}^{\beta} \varphi(t, \beta) d t d z \\
I_{40}(\alpha)=\int_{0}^{1} \sqrt{\psi_{0}(z, \alpha)} \int_{-1}^{z} \varphi(t, \alpha) d t d z, & I_{41}(\beta)=\int_{-1}^{1} \sqrt[4]{\psi_{1}(z, \beta)} \int_{-1}^{z} \varphi(t, \beta) d t d z \\
I_{5}(\alpha)=\int_{-1}^{\alpha} \varphi(t, \alpha) d t=\sqrt{\frac{2}{\alpha}} K\left(\sqrt{\frac{\alpha+1}{2 \alpha}}\right) & &
\end{array}
$$

where $K(\cdot)$ is the complete Legendre elliptic integral of the first kind.

In these notations from the paper of Kozlov and Vucans (1992) immediately follows:

Theorem 1 The effective coefficient of thermoconductivity $K_{\gamma, 0}$ of the structure $B_{\gamma, 0}$ for a fixed parameter $\gamma \in(0,1)$ is given by the two equivalent expressions

$$
K_{\gamma, 0}=\frac{1}{1+\gamma}\left[1+\frac{I_{30}(\alpha)}{I_{10}(\alpha) I_{5}(\alpha)}\right]=\frac{1}{1-\gamma^{2}}\left[1-\gamma \frac{I_{40}(\alpha)}{I_{20}(\alpha) I_{5}(\alpha)}\right]
$$

where $\alpha=\alpha(\gamma)$ is the unique solution from the interval $(1,+\infty)$ of the equation

$\gamma\left[I_{10}(\alpha)+I_{20}(\alpha)\right]=I_{20}(\alpha)$.

Moreover, from the papers of Kozlov and Vucans (1992) and Vucans (1992) follows:

\section{Theorem 2}

$$
\begin{gathered}
K_{\gamma, 0}=1+\left(1-\frac{\Gamma^{4}(1 / 4)}{8 \pi^{2}}\right) \gamma^{2}+o\left(\gamma^{2}\right) \approx 1-1.18844 \gamma^{2}+o\left(\gamma^{2}\right), \quad \text { when } \gamma \rightarrow 0_{+} \\
K_{\gamma, 0}=\frac{1}{2}+\frac{1}{2}\left(\frac{1}{2}+c_{1}\right)(1-\gamma)+\frac{1}{2}\left[\frac{1}{2}\left(\frac{1}{2}+c_{1}\right)+c_{2}\right](1-\gamma)^{2}+o\left((1-\gamma)^{2}\right) \approx \\
\approx 0.5+0.389682(1-\gamma)+0.230222(1-\gamma)^{2}+o\left((1-\gamma)^{2}\right), \quad \text { when } \gamma \rightarrow 1_{-},
\end{gathered}
$$

with

$c_{1}=\frac{4}{\pi^{2}} \int_{0}^{a r c s h 1} t \sqrt{\operatorname{csch}^{2} t-1} d t \approx 0.279364$

and

$c_{2}=-c_{1}\left\{\frac{1}{2 \pi}\left[5 \ln 2+4 \arctan \left(2^{-\frac{1}{4}}\right)\right]-\frac{5}{4}\right\} \approx 0.253296 c_{1} \approx 0.070762$.

We must note here that these asymptotic equalities coincide with results of Kozlov (1989) and Kozlov and Panasenko (1991).

Now using the method, similar as in the paper of Kozlov and Vucans (1992), we are going to prove the corresponding theorem for the structure $B_{\gamma, 1}$. 
Theorem 3 The effective coefficient of thermoconductivity $K_{\gamma, 1}$ of the structure $B_{\gamma, 1}$ for a fixed parameter $\gamma \in(0,1 / \sqrt{2})$ is given by the two equivalent expressions

$$
K_{\gamma, 1}=\frac{1-\sqrt{2} \gamma}{1-\gamma^{2}}\left[1+\frac{I_{31}(\beta)}{\sqrt{2} I_{11}(\beta) I_{5}(\beta)}\right]=\frac{1}{1-\gamma^{2}}\left[1-\frac{\gamma}{\sqrt{2}} \frac{I_{41}(\beta)}{I_{21}(\beta) I_{5}(\beta)}\right],
$$

where $\beta=\beta(\gamma)$ is the unique solution from the interval $(1,+\infty)$ of the equation

$\gamma I_{11}(\beta)=(1-\sqrt{2} \gamma) I_{21}(\beta)$.

Proof. First let's note that in our case $\theta=1$ the quarter $Q_{\gamma, 1}$ of the periodic structures unit cell $C_{\gamma, 1}$ is equal to the pentagon

$Q_{\gamma, 1}=\left\{\left(x_{1}, x_{2}\right) \in(0,1 / 2)^{2} \mid x_{1}+x_{2}<1-\gamma / \sqrt{2}\right\}$

with sides

$$
\begin{aligned}
& l_{1}=\left\{\left(x_{1}, x_{2}\right) \in R^{2} \mid x_{1}=0,0<x_{2}<1 / 2\right\}, \\
& l_{2}=\left\{\left(x_{1}, x_{2}\right) \in R^{2} \mid x_{2}=0,0<x_{1}<1 / 2\right\}, \\
& l_{3, \gamma}=\left\{\left(x_{1}, x_{2}\right) \in R^{2} \mid x_{1}=1 / 2,0<x_{2}<1 / 2-\gamma / \sqrt{2}\right\}, \\
& l_{4, \gamma}=\left\{\left(x_{1}, x_{2}\right) \in R^{2} \mid x_{1}+x_{2}=1-\gamma / \sqrt{2}, 1 / 2-\gamma / \sqrt{2}<x_{2}<1 / 2\right\}, \\
& l_{5, \gamma}=\left\{\left(x_{1}, x_{2}\right) \in R^{2} \mid x_{2}=1 / 2,0<x_{1}<1 / 2-\gamma / \sqrt{2}\right\} .
\end{aligned}
$$

Latter, without special references, we shall identify these sides of the boundary $\partial Q_{\gamma, 1}$ in $R^{2}$ with corresponding sets of points in the complex plane $C$. Due to the properties of periodicity of $\left(U_{\gamma, 1}-x_{1}\right)$ and the properties of symmetry of the set $C_{\gamma, 1}$ we can pass from the periodic boundary problem (1) on the unit cell $C_{\gamma, 1}$ to the "pure" third type boundary problem on its quarter $Q_{\gamma, 1}$ (for more details of this pass see, for example, the chapter 6 of the book of Bakhvalov and Panasenko (1989)):

$$
\begin{array}{lllll}
\Delta U_{\gamma, 1}=0 & \text { in } & Q_{\gamma, 1}, & U_{\gamma, 1}=0 \quad \text { in } l_{1}, \\
U_{\gamma, 1}=1 / 2 \quad \text { in } & l_{3, \gamma}, & \frac{\partial U_{\gamma, 1}}{\partial n_{Q}}=0 \quad \text { in } \quad l_{2} \cup l_{4, \gamma} \cup l_{5, \gamma},
\end{array}
$$

where $n_{Q}$ is the unit outward normal of the set $Q_{\gamma, 1}$. Moreover, from these properties of symmetry and from (2) and (5) by integration with respect to $x_{1}$ we get:

$$
\begin{aligned}
K_{\gamma, 1} & =\frac{1}{m e s Q_{\gamma, 1}} \iint_{Q_{\gamma, 1}} \frac{\partial U_{\gamma, 1}\left(x_{1}, x_{2}\right)}{\partial x_{1}} d x_{1} d x_{2}= \\
& =\frac{4}{1-\gamma^{2}}\left[\frac{1}{4}(1-\sqrt{2} \gamma)+\int_{1 / 2-\gamma / \sqrt{2}}^{1 / 2} U_{\gamma, 1}\left(1-\gamma / \sqrt{2}-x_{2}, x_{2}\right) d x_{2}\right]= \\
& =\frac{1}{1-\gamma^{2}}\left[1-\sqrt{2} \gamma+\frac{4}{i-1} \int_{l^{4}, \gamma} U_{\gamma, 1}(\xi) d \xi\right],
\end{aligned}
$$


where the last integral is presented in terms of the complex variable $\xi$ along the side $l_{4, \gamma}$ of the boundary $\partial Q_{\gamma, 1}$ from point $(1 / 2+i(1 / 2-\gamma / \sqrt{2}))$ to point $(1 / 2-\gamma / \sqrt{2}+i / 2)$.

In order to obtain the solution $U_{\gamma, 1}$ of the problem (5) we construct the conformal representation of the upper half plane to $Q_{\gamma, 1}$. According to Schwarz-Christoffel theorem such one-to-one mapping $\{z: \operatorname{Im} z>0\} \stackrel{\omega}{\longmapsto}\left\{\xi: \xi \in Q_{\gamma, 1}\right\}$ is given by

$\xi=\omega(z)=(i-1) c_{0} \int_{0}^{z} \frac{d t}{\sqrt[4]{1-t^{2}} \sqrt{\beta^{2}-t^{2}}}+\frac{1+i}{2 \sqrt{2}}(\sqrt{2}-\gamma)$

where $\beta$ satisfies (4) and for the real constant $c_{0}$ we have equivalent expressions:

$c_{0}=\frac{\gamma}{2 \sqrt{2} I_{21}(\beta)}=\frac{1-\sqrt{2} \gamma}{2 \sqrt{2} I_{11}(\beta)}$.

The mapping $\omega$ transforms the real axis into the boundary $\partial Q_{\gamma, 1}$ in the following way:

$\begin{array}{lll}\omega((-\infty,-\beta))=l_{2}, & \omega((-\beta,-1))=l_{3, \gamma}, & \omega((-1,1))=l_{4, \gamma}, \\ \omega((1, \beta))=l_{5, \gamma}, & \omega((\beta,+\infty))=l_{1} . & \end{array}$

We remark here that equality (4) defines for $\beta>1$ the strictly decreasing function $\gamma=$ $\gamma(\beta)$. This function has the following limiting values: $\gamma\left(1_{+}\right)=1 / \sqrt{2}, \gamma(+\infty)=0$. So, in the interval $(0,1 / \sqrt{2})$ there exists a strictly decreasing inverse function $\beta=\beta(\gamma)$.

Now let's introduce the complex function

$\Phi(z)=c_{1}\left[\int_{0}^{z} \frac{d t}{\sqrt{(1+t)\left(\beta^{2}-t^{2}\right)}}+c_{2}\right]$

of the complex variable $z=z_{1}+i z_{2}\left(z_{1}, z_{2} \in R\right)$ where $c_{1}$ and $c_{2}$ are real constants:

$c_{1}=\frac{-1}{2 I_{5}(\beta)}, \quad c_{2}=-\int_{0}^{\beta} \varphi(t, \beta) d t$

For points $z$ in the upper half plane and on the real axis the integrals in (7) and (10) are defined along any path from 0 to $z$ which completely lies in the upper half plane.

It's easy to verify the following properties of the function $\Phi$ :

(i) $\operatorname{Re} \Phi$ is harmonic in the upper half plane;

(ii) $\operatorname{Re} \Phi$ has the following limiting values on the real axis:

$$
\begin{array}{lll}
\operatorname{Re} \Phi(z)=-c_{1} \int_{-\infty}^{z} \varphi(t, \beta) d t & \text { if } & z \in(-\infty,-\beta] \\
\operatorname{Re} \Phi(z)=1 / 2 & \text { if } & z \in[-\beta,-1] \\
\operatorname{Re} \Phi(z)=-c_{1} \int_{z}^{\beta} \varphi(t, \beta) d t & \text { if } & z \in[-1, \beta] \\
\operatorname{Re} \Phi(z)=0 & \text { if } & z \in[\beta,+\infty)
\end{array}
$$

(iii) in the intervals $(-\infty,-\beta)$ and $(-1, \beta)$ on the real axis the derivative $\frac{\partial \Phi\left(z_{1}+i z_{2}\right)}{\partial z_{2}}$ is purely imaginary, therefore $\frac{\partial R e \Phi\left(z_{1}+i z_{2}\right)}{\partial z_{2}}=0$ there. 
From the properties i), ii), iii) and from (7) follows that the solution of the problem (5) is equal to

$U_{\gamma, 1}(\xi)=\operatorname{Re} \Phi\left(\omega^{-1}(\xi)\right)$

From the property ii) changing the variable and taking into account (7), (8), (9) and (11) we get:

$$
\begin{aligned}
\int_{l_{1, \gamma}} U_{\gamma, 1}(\xi) d \xi & =\int_{l_{1, \gamma}} \operatorname{Re} \Phi\left(\omega^{-1}(\xi)\right) d \xi=\int_{-1}^{1} \operatorname{Re} \Phi(z) \omega^{\prime}(z) d z= \\
& =-c_{0} c_{1}(i-1) \int_{-1}^{1} \frac{1}{\sqrt[4]{1-z^{2}} \sqrt{\beta^{2}-t^{2}}} \int_{z}^{\beta} \frac{d t}{\sqrt{(1+t)\left(\beta^{2}-t^{2}\right)}}= \\
& =\frac{(i-1)(1-\sqrt{2} \gamma) I_{31}(\beta)}{4 \sqrt{2} I_{11}(\beta) I_{5}(\beta)} .
\end{aligned}
$$

This formula jointly with (6), (8) and (11) gives the result of Theorem 3.

The obtained formulas (3) allow us to make the asymptotic analysis of the $K_{\gamma, 1}$ in the cases when in our periodic structure the squareic holes are small $\left(\gamma \rightarrow 0_{+}\right)$or when they are in the position near to touch one another $\left(\gamma \rightarrow 1 / \sqrt{2}_{-}\right)$.

\section{Theorem 4}

$$
\begin{aligned}
& K_{\gamma, 1}=1+\left(1-\frac{\Gamma^{4}(1 / 4)}{8 \pi^{2}}\right) \gamma^{2}+o\left(\gamma^{2}\right) \approx 1-1.18844 \gamma^{2}+o\left(\gamma^{2}\right), \text { when } \gamma \rightarrow 0_{+} \\
& K_{\gamma, 1}=-\frac{\pi}{2} \frac{1}{\ln (1 / \sqrt{2}-\gamma)}+o\left(\frac{1}{\ln (1 / \sqrt{2}-\gamma)}\right), \quad \text { when } \gamma \rightarrow 1 / \sqrt{2} 2_{-}
\end{aligned}
$$

Proof. a) Let $\gamma \rightarrow 0_{+}$; in this case from (4) and from our definitions we have:

$$
\begin{array}{ll}
\lim _{\beta \rightarrow+\infty} \sqrt{\beta} I_{11}(\beta)=\frac{\Gamma^{2}(1 / 4)}{2 \sqrt{2 \pi}}, & \lim _{\beta \rightarrow+\infty} \beta I_{21}(\beta)=\frac{(2 \pi)^{3 / 2}}{\Gamma^{2}(1 / 4)}, \\
\lim _{\beta \rightarrow+\infty} \sqrt{\beta} \gamma(\beta)=\frac{8 \pi^{2}}{\Gamma^{4}(1 / 4)}, & \lim _{\gamma \rightarrow 0_{+}} \beta(\gamma)=+\infty, \\
\lim _{\beta \rightarrow+\infty} \beta^{2} I_{41}(\beta)=\sqrt{2} \pi, & \lim _{\beta \rightarrow+\infty} \sqrt{\beta} I_{5}(\beta)=\frac{\Gamma^{2}(1 / 4)}{2 \sqrt{2 \pi}},
\end{array}
$$

The asymptotic equality (12) follows from these properties.

b) Let $\gamma \rightarrow 1 / \sqrt{2_{-}}$; in this case from (4) and from our definitions we have:

$$
\begin{array}{ll}
\lim _{\beta \rightarrow 1_{+}} \frac{I_{11}(\beta)}{\sqrt[4]{\beta-1}}=\frac{2^{7 / 4} \pi^{3 / 2}}{\Gamma^{2}(1 / 4)}, & \lim _{\beta \rightarrow 1_{+}} I_{21}(\beta)=\frac{\Gamma^{2}(1 / 4)}{2 \sqrt{2 \pi}}, \\
\lim _{\beta \rightarrow 1_{+}} \frac{1 / \sqrt{2}-\gamma}{\sqrt[4]{b-1}}=\frac{2^{9 / 4} \pi^{2}}{\Gamma^{4}(1 / 4)}, & \lim _{\gamma \rightarrow 1 / \sqrt{2}_{-}} \beta(\gamma)=1_{+},
\end{array}
$$




$$
\lim _{\beta \rightarrow 1_{+}} I_{31}(\beta)=\frac{\sqrt{\pi}}{2} \Gamma^{2}(1 / 4), \quad \quad \quad \lim _{\beta \rightarrow 1_{+}} \frac{I_{5}(\beta)}{\ln (\beta-1)}=-\frac{1}{\sqrt{2}} .
$$

The asymptotic equality (13) follows from these properties.

We note that in the case of small holes $\left(\gamma \rightarrow 0_{+}\right)$the asymptotic formulas for $K_{\gamma, 0}$ and $K_{\gamma, 1}$ are equal up to the terms with $\gamma^{2}$. This agrees with the result of Kozlov (1989).

\section{REFERENCES}

Bakhvalov, N. and Panasenko, G.P. (1989) Homogenization: averaging processes in periodic media. Dordrecht/Boston/London: Kluwer Academic Publishers.

Bensoussan, A., Lions, J.-L. and Papanicolaou, G. (1978) Asymptotic analysis for periodic structures. Amsterdam, North-Holland Publ. Comp.

Kozlov, S.M. (1989) Geometric aspects of homogenization. Russian Mathematical Surveys, 44, 2, 91-144.

Kozlov, S.M. and Panasenko, G.P. (1991) Corrections to the strength materials theory for the lattice structure. Publication of the Laboratory of Numerical Analysis of the University Paris-VI, R91020, 9p.

Kozlov, S.M. and Vucans, J. (1992) Explicit formulæ for effective thermoconductivity on the squareic lattice structure. Comptes Rendus de l'Académie des Sciences, I, 314, 281-6.

Vucans, J. (1992) Approximation of the third order to effective thermoconductivity of the squareic lattice structure. Acta Universitatis Latviensis, 575, 125-32. 